Adv. Radio Sci., 5, 447-450, 2007

www.adv-radio-sci.net/5/447/2007/

(C) Author(s) 2007. This work is licensed

Advances in

under a Creative Commons License.

Radio Science

\title{
On the statistics of differential phases in C-band weather radar
}

\author{
T. Otto and M. Chandra \\ Professorship of Microwave Engineering and Photonics, Chemnitz University of Technology, Germany
}

\begin{abstract}
Differential phases open a new parameter in polarimetric weather radar data analysis. In polarimetric Smatrices measured at the linear polarisation bases (vertical and horizontal) there exist basically three differential phases namely the differential propagation phase and two co-to-crosspolar phases. In this paper we shall investigate the statistics of differential propagation phase and co-tocrosspolar phases in weather radar data recorded with the Cband weather radar of DLR, Wessling.
\end{abstract}

\section{Introduction}

\subsection{Polarimetric S-matrix measurement}

The POLDIRAD is DLR's fully polarimetric Doppler weather radar. It is able to measure the fully polarimetric $\mathbf{S}$-matrix at the linear polarisation bases horizontal and vertical

$\left(\begin{array}{c}E_{v}^{r} \\ E_{h}^{r}\end{array}\right)=\left(\begin{array}{c}S_{v v}(t) S_{v h}(t+T) \\ S_{h v}(t) S_{h h}(t+T)\end{array}\right)\left(\begin{array}{c}E_{v}^{t} \\ E_{h}^{t}\end{array}\right) \frac{e^{-j k 2 R_{N}}}{R_{N}^{2}}$

where $E_{v / h}^{r}$ are the backscattered and $E_{v / h}^{t}$ are the transmitted electrical fields and $T$ is the pulse repetition time. $R_{N}$ is the range between the radar and the near edge of the radar pulse volume and $k$ is the wave number.

As could be seen, the two columns of the polarimetric S-matrix are determined by two consecutive measurements. The POLDIRAD transmits alternately vertical and horizontal polarised pulses and receives the backscattered echoes simultaneously in these two orthogonal polarised channels. See Schroth et al. (1988) for a further discussion of the measurement scheme of the POLDIRAD.

Correspondence to: T. Otto

(tobias.otto@etit.tu-chemnitz.de)
Figure 1 shows the radar geometry. For each radar pulse volume there is a measurement of $M$ polarimetric $\mathbf{S}$-matrices where $M$ is the sample size.

It is possible to define several differential phases within the measured $\mathbf{S}$-matrices. The first is the differential propagation phase $\Psi_{d p}$ which is the phase difference between the two copolar $\mathbf{S}$-matrix elements $S_{h h}$ and $S_{v v}$. The co-to-crosspolar phases $\Psi_{c r, h}$ and $\Psi_{c r, v}$ are the phase differences between a copolar and a crosspolar S-matrix element.

\subsection{Example weather radar dataset}

Figure 2 shows an example of weather radar data used within this paper. To obtain these so called RHI (range height indicator) scans the measurement was done while moving the radar antenna only along the elevation.

Figure 2a shows the reflectivity $Z_{h h}$ :

$Z_{h h}=10 \log _{10}\left(C R_{N}^{2} P_{h h}\right)(d B Z)$

where $C$ is the radar constant and $P_{h h}$ is the power of the horizontal polarised channel.

Another very common polarimetric weather radar measurement quantity is the differential reflectivity $Z_{d r}$ in Fig. 2b:

$Z_{d r}=10 \log _{10} \frac{P_{h h}}{P_{v v}}(d B)$.

\section{The differential propagation phase}

2.1 Physical background of the differential propagation phase

The differential propagation phase $\Phi_{d p}$ is the two way phase difference between the vertical and horizontal polarised pulse. It is the only measurement quantity in radar meteorology that contains information about the forward scattering of

Published by Copernicus Publications on behalf of the URSI Landesausschuss in der Bundesrepublik Deutschland e.V. 
Table 1. Standard Deviation of $\Psi_{d p, 2}$ for $M=32,64$ and 128 in dependence of $\left|\rho_{h v}\right|$.

\begin{tabular}{lcccc}
\hline & $1.0 \geq\left|\rho_{h v}\right|>0.95$ & $0.95 \geq\left|\rho_{h v}\right|>0.9$ & $0.9 \geq\left|\rho_{h v}\right|>0.85$ & $0.85 \geq\left|\rho_{h v}\right|>0.8$ \\
\hline $\mathrm{SD}\left(\Psi_{d p, 2}\right)$ for $M=32$ & $2.75^{\circ}$ & $6.33^{\circ}$ & $7.54^{\circ}$ & $9.76^{\circ}$ \\
$\mathrm{SD}\left(\Psi_{d p, 2}\right)$ for $M=64$ & $2.12^{\circ}$ & $4.67^{\circ}$ & $5.53^{\circ}$ & $7.05^{\circ}$ \\
$\mathrm{SD}\left(\Psi_{d p, 2}\right)$ for $M=128$ & $1.63^{\circ}$ & $3.42^{\circ}$ & $4.03^{\circ}$ & $5.11^{\circ}$ \\
\hline
\end{tabular}

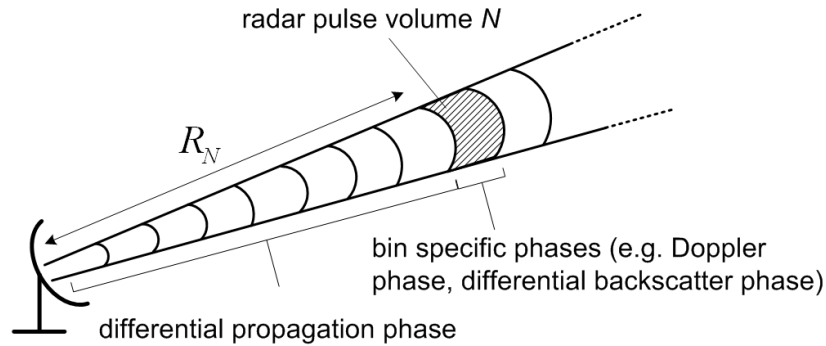

$\Phi_{d p}=\arg \left[e^{-j\left(K_{h h}-K_{v v}^{*}\right) R_{N}}\right]$

Fig. 1. Radar geometry.

the electromagnetic waves and its relationship to the rain rate is nearly linear at C-band, cf. Scarchilli et al. (1993).

$\Phi_{d p}$ is defined by

$\Phi_{d p}=\arg \left[e^{-j\left(K_{h h}-K_{v v}^{*}\right) R_{N}}\right]$

where $K_{h h}$ and $K_{v v}$ are the effective external two-way complex propagation constants for horizontal and vertical polarisation.

\subsection{Calculation of the differential propagation phase}

The term $\arg \left\langle S_{h h} S_{v v}^{*}\right\rangle$ contains the differential propagation phase $\Phi_{d p}$ but also a Doppler phase shift because there is a time delay of the pulse repetition time $T$ between the measurements of the two columns of the polarimetric S-matrix. This Doppler phase shift needs to be removed for a proper estimation of differential propagation phase. To achieve that there are the two following ways:

$\Psi_{d p, 1}=\arg \left\langle S_{h h}(t+T) S_{v v}^{*}(t)\right\rangle-\frac{\arg \left\langle S_{v v}^{*}(t) S_{v v}^{*}(t+2 T)\right\rangle}{2}$

$\Psi_{d p, 2}=$

$\frac{\arg \left\langle S_{v v}^{*}(t) S_{h h}(t+T)\right\rangle+\arg \left\langle S_{h h}(t+T) S_{v v}^{*}(t+2 T)\right\rangle}{2}$

where \langle\rangle denotes the average over $M$ consecutive samples obtained of each radar pulse volume and ${ }^{*}$ means the complex conjugate.

After removing the Doppler phase shift the resulting phase still contains several bin specific phases namely the differential backscatter phase and the internal propagation phase shift
(Chandra, 1991). From now on $\Psi_{d p}$ will be used as symbol for the differential propagation phase because it is not possible to separate the bin specific phases from the real differential propagation phase $\Phi_{d p}$ which is the range dependent phase part. In most cases the bin specific phases are negligible and the calculated $\Psi_{d p}$ is equal to $\Phi_{d p}$.

\subsection{Statistics of the differential propagation phase}

The standard deviation of the estimation of differential propagation phase depends on the sample size $M$, the Doppler spectrum width and the correlation between the two copolar S-matrix elements (Sachidananda and Zrnic, 1986).

The correlation between the two copolar S-matrix elements is given by the magnitude of the copolar correlation coefficient:

$\left|\rho_{h v}\right|=\frac{\left|\left\langle S_{h h} S_{v v}^{*}\right\rangle\right|}{\sqrt{\left\langle\left|S_{h h}\right|^{2}\right\rangle\left\langle\left|S_{v v}\right|^{2}\right\rangle}}$.

This correlation is very high for weather targets at the polarisation basis vertical and horizontal. This high correlation between the two copolar S-matrix elements is necessary for a good estimation of differential propagation phase. The lower $\left|\rho_{h v}\right|$ the higher is the standard deviation of the estimated $\Psi_{d p}$. Figure $2 \mathrm{c}$ shows the RHI of $\left|\rho_{h v}\right|$ with it's very high values except in the region of the melting layer at a height of $2-3 \mathrm{~km}$ and in the upper right corner due to a very bad signal-to-noise ratio (SNR) there.

The magnitude of the copolar correlation coefficient depends on several microphysical properties of the hydrometeors within the radar pulse volume such as particle shape variations or canting angle of the hydrometeors but it also depends on the time delay between the measurement of the two columns of the $\mathbf{S}$-matrix (Chandra et al., 1992). If this time difference is greater than the decorrelation time of hydrometeors then the correlation between the two copolar $\mathbf{S}$-matrix elements is very low.

Figure 3 shows the dependence of the standard deviation of the differential propagation phase $\Psi_{d p, 2}$ against the sample size $M$ and the magnitude of the copolar correlation coefficient. This plot is obtained using one of POLDIRADs time series dataset from a stratiform storm. Table 1 contains some values of the standard deviation of the differential propagation phase for sample sizes of 32, 64 and 128 .

As predicted by the theory the standard deviation of the differential propagation phase is lower the more samples are 

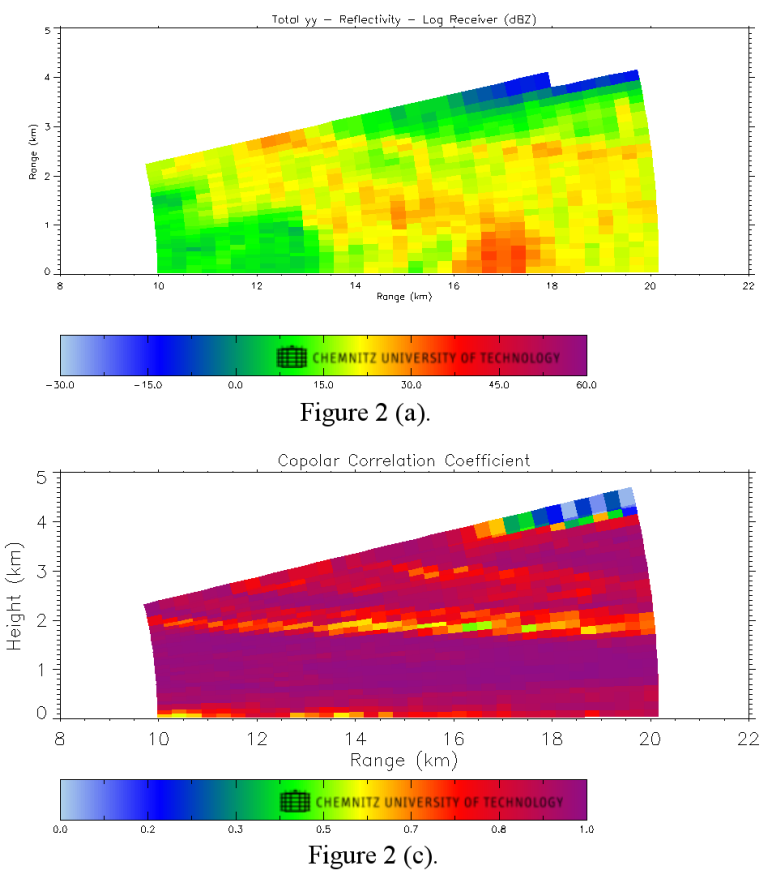

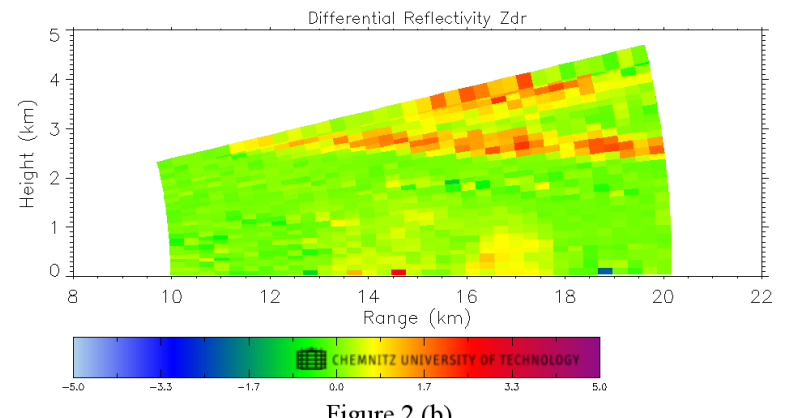

Figure 2 (b).

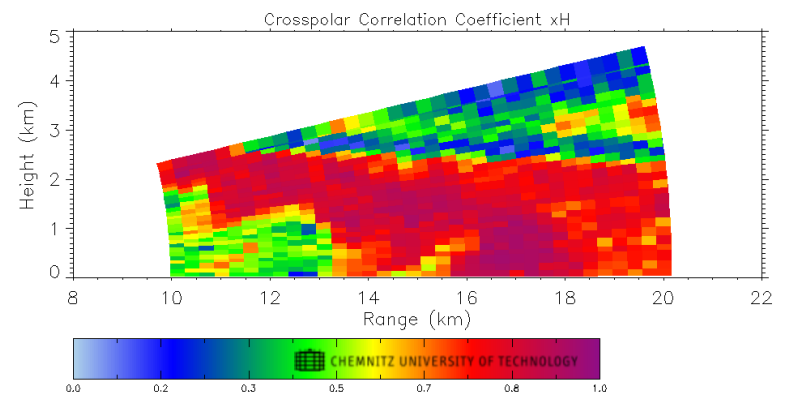

Figure 2 (d)

Fig. 2. (a) Reflectivity $Z_{h h}(d B Z)$, (b) Differential Reflectivity $Z_{d r}(d B)$, (c) Magnitude of the copolar correlation coefficient $\left|\rho_{h v}\right|$, (d) Magnitude of the co-to-crosspolar correlation coefficient $\left|\rho_{c r, h}\right|$ of a weather radar dataset recorded with the POLDIRAD in autumn 1994.

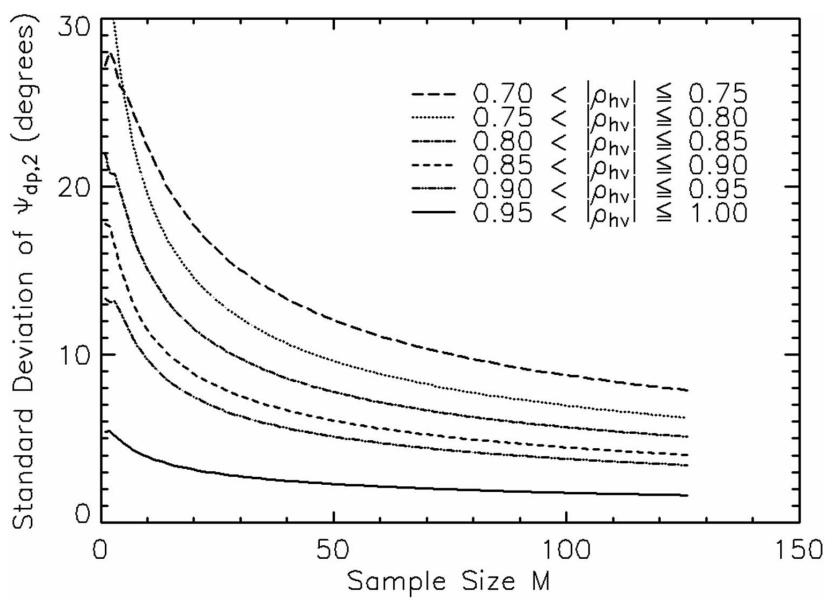

Fig. 3. Standard deviation of differential propagation phase $\Psi_{d p, 2}$ in dependence of the sample size $M$ and the magnitude of the copolar correlation coefficient $\left|\rho_{h v}\right|$.

measured and the higher the magnitude of the copolar correlation coefficient is. The differential propagation phase could be determined with an accuracy of $1-2^{\circ}$ for $\left|\rho_{h v}\right|$ greater than 0.95 by DLR's POLDIRAD. These values of the standard deviation of the differential propagation phase were also confirmed by other datasets of the POLDIRAD.

This result for the standard deviation of the differential propagation phase obtained from data corresponds to values obtained by simulation for C-Band, e.g. Hubbert et al. (1993).

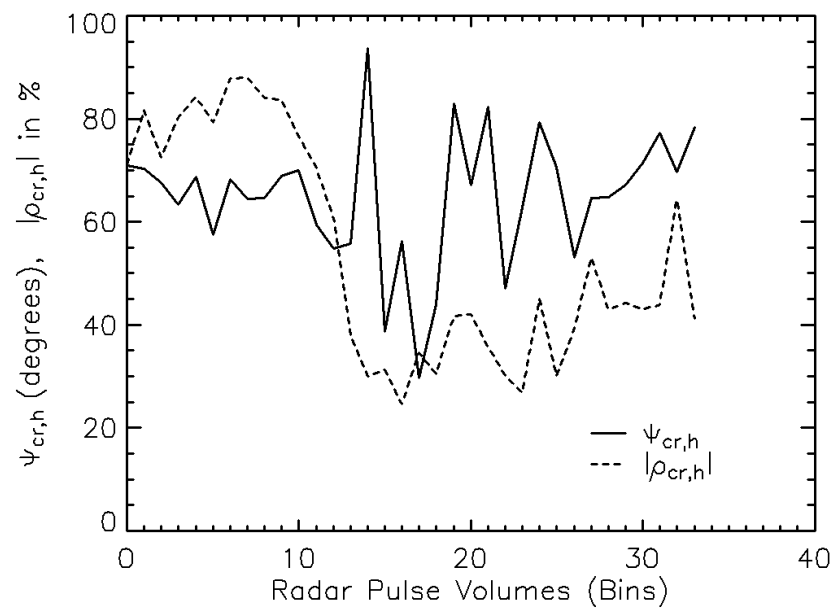

Fig. 4. Rayplot of $\Psi_{c r, h}$ and $\left|\rho_{c r, h}\right|$ for an elevation of $10.9^{\circ}$.

\section{The co-to-crosspolar phases}

\subsection{Calculation of the co-to-crosspolar phases}

The differential phases between a copolar and a crosspolar S-matrix element are named the co-to-crosspolar phases:

$\Psi_{c r, h}=\arg \left\langle S_{v h} S_{h h}^{*}\right\rangle$,

$\Psi_{c r, v}=\arg \left\langle S_{h v} S_{v v}^{*}\right\rangle$.

The co-to-crosspolar phases do not contain a Doppler phase shift because the copolar and the crosspolar re- 
turns are measured simultaneously by two receivers in the POLDIRAD. They also consist of a range dependent phase and a bin specific phase.

The co-to-crosspolar phases will only deliver some results if a crosspolar signal is present and thus depolarisation must have occurred, e.g. by the presence of canted hydrometeors.

\subsection{Statistics of the co-to-crosspolar phases}

The statistics of co-to-crosspolar phases will only be treated qualitatively in this paper. Therefore, Fig. 4 shows a ray plot of co-to-crosspolar phase $\Psi_{c r, h}$ and of the magnitude of the co-to-crosspolar correlation coefficient $\left|\rho_{c r, h}\right|$ :

$\left|\rho_{c r, h}\right|=\frac{\left|\left\langle S_{v h} S_{h h}^{*}\right\rangle\right|}{\sqrt{\left\langle\left|S_{v h}\right|^{2}\right\rangle\left\langle\left|S_{h h}\right|^{2}\right\rangle}}$.

It is seen that low $\left|\rho_{c r, h}\right|$ values results in large deviations of the co-to-crosspolar phase, i.e. starting at bin 13, which is the region of the melting layer.

The problem with co-to-crosspolar phases is the low correlation between the copolar and the crosspolar $\mathbf{S}$-matrix element at the linear polarisation bases horizontal and vertical, Fig. 2d. This leads especially in the melting layer to very noisy estimates of the co-to-crosspolar phases.

\section{Conclusions}

The differential propagation phase could be determined up to an accuracy of $1-2^{\circ}$ by DLR's POLDIRAD for the linear polarisation bases horizontal and vertical. Not solved yet is the problem of the discrimination of the range dependent differential propagation phase and the bin specific phases, i.e. mainly the differential backscatter phase.
Due to the bad correlation of copolar to crosspolar returns for the linear polarisation bases (horizontal and vertical) the quality of estimation of co-to-crosspolar phase is very noisy. For that reason it is difficult to find useful applications for the co-to-crosspolar phases. Nevertheless, the behaviour of co-to-crosspolar phases at the linear polarisation bases horizontal and vertical needs more attention in research.

This paper points out that contrary to common belief observable co-to-crosspolar phases do exist and exhibit a physical signature, even though they are noisy.

\section{References}

Chandra, M.: Prediction of Propagation Effects and Rain-Intensity using Radar Determined Three-Parameter Raindrop-Size Distribution, ICAP-91, York, UK, IEE Publ. 333, 1, 88-94, 1991.

Chandra, M., Clemens, E., Ritenberg, F., Schnabl, G., Schroth, A., and Tragl, K.: DFVLR Polarimetric Meteorological Instrumentation Radar Facility, in: Direct and Inverse Methods in Radar Polarimetry, edited by: W.-M. Boerner et al., Kluwer Academic Publishers, 1579-1612, 1992.

Hubbert, J., Chandrasekar, V., and Bringi, V. N.: Processing and Interpretation of Coherent Dual-Polarized Radar Measurements, Journal of Atmospheric and Oceanic Technology, 10, 155-164, 1993.

Sachidananda, M. and Zrnic, D. S.: Differential propagation phase shift and rainfall rate estimation, Radio Science, 21, 2, 235-247, 1986.

Scarchilli, G., Gorgucci, E., Chandrasekar, V., and Seliga, T. A.: Rainfall Estimation Using Polarimetric Techniques at C-Band Frequencies, Journal of Applied Meteorology, 32, 1150-1160, 1993.

Schroth, A. C., Chandra, M. S., and Meischner, P. F.: A C-Band coherent polarimetric radar for propagation and cloud physics research, Journal of Atmospheric and Oceanic Technology, 5, 803-822, 1988. 